\title{
El incremento del indice de masa corporal materno se relaciona con aumento del riesgo de resultados adversos perinatales
}

\author{
Maternal body mass index rise is related to an increased risk of adverse perinatal outcomes
}

Objetivo

Esclarecer la asociación entre el índice de masa corporal (IMC) materno y el riesgo de muerte fetal, muerte al nacer y muerte infantil.

\section{Fuente de datos}

PubMed y Embase desde su origen hasta el 23 de enero de 2014.

\section{Selección de estudios y extracción de datos}

Se incluyeron estudios de cohorte que reportaron el IMC materno en el embarazo temprano y el riesgo de muerte fetal, aborto, mortinato, muerte neonatal, perinatal o infantil. Se incluyeron publicaciones que estimaron el riesgo relativo (RR) ajustado a tres o más categorías de IMC. Se utilizó un modelo
Aune D. y col. JAMA. 2014;311(15):1536-46. de efectos aleatorios, y se calcularon los RR con sus IC 95\%: en relación al incremento de cinco puntos del IMC y se exploró la asociación con el valor del IMC en forma continua.

\section{Resultados principales}

Fueron incluidos 38 estudios, cuya calidad en general fue alta. No se observó variabilidad de los resultados de acuerdo a la calidad de los mismos. Los principales resultados se resumen en la tabla 1. El análisis de sensibilidad mostró robustez de los hallazgos, entre ellos el análisis de los estudios en los cuales se excluyeron las pacientes con factores de alto riesgo (hipertensión arterial, diabetes pregestacional, preeclampsia y anomalías congénitas).

Tabla 1. Riesgo de eventos obstétricos adversos en relación con el índice de masa corporal materno.

\begin{tabular}{c|c|c|c|c|} 
Variable & $\mathbf{N}^{\circ}$ estudios & Casos/Mujeres embarazadas & RR\# (IC95\%) & Relación con el IMC \\
Muerte fetal & 7 & $10.147 / 690.622$ & $1,21(1,09 \mathrm{a} 1,35)$ & no lineals \\
Muerte al nacer & 18 & $16.274 / 3.288 .688$ & $1,24(1,18 \mathrm{a} 1,30)$ & casi lineal \\
Muerte perinatal & 11 & $4.311 / 982.236$ & $1,16(1,00 \mathrm{a} 1,35)$ & no lineal \\
Muerte neonatal y pos-neonatal & 12 & $11.294 / 3.321 .555$ & $1,15(1,07 \mathrm{a} 1,23)^{*}$ & no lineal \\
Muerte Infantil & 4 & $4.983 / 1.491 .879$ & $1,18(1,09 \mathrm{a} 1,28)$ & no lineal \\
\hline
\end{tabular}

\#Por cada cinco unidades de incremento en el IMC. §pronunciada, especialmente con IMC mayor de 40. *Riesgo de sesgo de publicación (valor de $p$ de la prueba de Egger 0,02). ¥RR mayor con muerte neonatal temprana. IMC: índice de masa corporal.

\section{Conclusiones}

Incrementos modestos del IMC materno se asocian con riesgo aumentado de muerte fetal, neonatal, perinatal e infantil. Las guías para el control del peso en mujeres que planean embara- zos deberían tomar estos hallazgos en consideración.

Fuentes de financiamiento/conflicto de interés: Fundación Noruega de síndrome de muerte súbita infantil y muerte al nacer.

\section{Comentario}

La obesidad tiene proporciones epidémicas en algunos países y alrededor del mundo. La obesidad materna se convirtió en la actualidad en uno de los principales factores de riesgo generales en la práctica obstétrica. Distintos estudios epidemiológicos muestran una asociación entre la obesidad y un aumento en la tasa de abortos espontáneos, anomalías fetales congénitas, tromboembolismo, diabetes gestacional, pre-eclampsia, y hemorragia postparto, muerte fetal y neonatal, entre otros resultados obstétricos adver$\operatorname{sos}^{1}$. Por ejemplo, se observa un aumento de tres veces el riesgo de muerte fetal (OR 2,79; IC 95\% 1,94 a 4,02) en pacientes obesas mórbidas comparadas con embarazadas con un IMC menor de $20^{2}$. El presente meta-análisis muestra la asociación entre el IMC materno y el riesgo de muerte fetal y neonatal, encontrándose una asociación entre el aumento de peso y el aumento del riesgo de muerte fetal. Sin embargo, el metanálisis muestra heterogeneidad en sus resultados, probablemente atribuible a las diferencias en las poblaciones estudiadas, la cual inevitablemente afecta las conclusiones. Esta es una de las mayores limitaciones del estudio, ya que muchos de los resultados reportados pueden deberse a las diferentes comorbilidades encontradas en mujeres obesas (ejemplo, pre-eclampsia, diabetes), o simplemente a heterogeneidad estadística. Estudios diseñados controlando por confundidores, brindarían probablemente mejores conclusiones respecto a la asociación entre obesidad materna y los resultados neonatales. Por otra parte, el embarazo y el postparto representan una ventana única debido a la exposición repetida de la mujer al sistema de salud, otorgando la oportunidad para realizar intervenciones que permitan beneficiar la salud futura de las mujeres y sus hijos. Debido a estas características, sería importante que el paquete de intervenciones contenidas en el control prenatal contemplaran no solo la perspectiva gestacional, sino la salud a largo plazo. Tanto la obesidad como la ganacia de peso excesiva durante el embarazo son factores de riesgo modificables a través de intervenciones que modifican el estilo de vida con claros beneficios para la mujer y su familia ${ }^{3}$.

\section{Conclusión del comentador}

La evidencia actual sugiere que aún los más mínimos incrementos del IMC materno tienen una repercusión en los resultados neonatales, incrementando el riesgo de muerte fetal, al nacer, neonatal, perinatal e infantil. El IMC óptimo para prevenir estos eventos aún es desconocido. Sin embargo, estos resultados deben ser tenidos en cuenta en las actualizaciones de las guías de manejo del peso corporal en mujeres embarazadas con el fin de reducir el número de muertes fetales, perinatales e infantiles.

\footnotetext{
Gustavo Izbizky [ SubJefe del Servicio de Obstetricia del Hospital Italiano de Buenos Aires ]

y María Lucrecia Absi [ Residente de Tocoginecología del Hospital Italiano de Buenos Aires. gustavo.izbizky@hospitalitaliano.org.ar ]
}

Resumido por Franco J, y comentado por Izbizky G, Absi ML. El incremento del índice de masa corporal materno se relaciona con aumento del riesgo de resultados adversos perinatales. Evid Actual Pract Ambul. 2015;18(3):83. Jul-Sep. Comentado de: Aune D, y col. Maternal Body Mass Index and the Risk of Fetal Death, Stillbirth, and Infant Death. A Systematic Review and Meta-analysis. JAMA. 2014;311(15):1536-46. PMID: 24737366.

\section{Referencias}

1. CMACE- RCOG Joint Guideline. Management of Women with Obesity in Pregnancy. 2010. Disponible en: https:/www.rcog.org.uk/globalassets/documents/guidelines/cmacercogjointguidelinemanagementwomenobesitypregnancya.pdf

2. Davies G. Obesity in Pregnancy. J Obstet Gynaecol Can 2010;32:165-173.

3. Gayle O, Blackwell S. Optimization of Gestational Weight Gain in the Obese Gravida: A Review. Obstet Gynecol Clin N Am 2011;38: 397-407. 